\title{
A STUDY ON THE SECURE CODING RULES FOR DEVELOPING SECURE SMART CONTRACT ON ETHEREUM ENVIRONMENTS
}

\author{
Junho Jeong ${ }^{1}$, Yunsik Son ${ }^{2, *}$ and Yangsun Lee ${ }^{3, *}$ \\ ${ }^{l}$ Dept. of Computer Science and Engineering, Kongju National University, \\ Cheonan, Korea \\ ${ }^{2}$ Dept. of Computer Science and Engineering, Dongguk University, Seoul, Korea \\ ${ }^{3}$ Dept. of Computer Engineering, Seokyeong University, Seoul, Korea \\ ${ }^{1}$ yanyenli@kongju.ac.kr, ${ }^{2 *}$ sonbug@dongguk.edu, ${ }^{3 *}$ yslee@ skuniv.ac.kr
}

\begin{abstract}
Smart contract-based development of decentralized applications is increasing with the development of blockchain technology. Although blockchainbased smart contracts are expected to revolutionize the digital economy, several security issues need to be addressed before this technology can be used reliably. The recent discovery of security weaknesses in Ethereum smart contracts questions the reliability of smart contracts. Therefore, there is a need to create and diagnose security weaknesses in Ethereum smart contracts to mitigate security risks. In this study, we assessed the potential security weaknesses of running smart contracts on Ethereum.
\end{abstract}

Keywords - Secure Coding; Smart Contract; blockchain; Software Weakness; Ethereum

\section{INTRODUCTION}

The use of smart contracts is increasing with the development of using blockchain technology. Although blockchain-based smart contracts are expected to revolutionize the digital economy, several security issues need to be addressed before this technology can be used reliably. One such example is the recent decentralized autonomous organization (DAO) and Parity hacking incidents, which has diminished trust in smart contracts, in Ethereum [1-4]. Therefore, there is a need to create and inspect security weaknesses in Ethereum smart contracts to prevent security risks. This study proposes potential security weaknesses that may occur when running smart contracts on Ethereum. By diagnosing the security weaknesses proposed at the time of decentralized application (DApp) development and mitigating them in advance, developers can expect to increase the security of smart contracts.

\section{RELATED STUDIES}

\subsection{SECURIFY}

SECURIFY is a result of the ETH Zurich blockchain security project in 2017 and is an Ethereum smart contract diagnostic tool [5,6]. SECURIFY diagnoses smart contracts using automated analysis techniques such as static and dynamic analysis to prevent

Received: October 8, 2019

Reviewed: December 2, 2019

Accepted: December 11, 2019

${ }^{*}$ Corresponding Author 
security risks. Table I shows SECURIFY's smart contract security weakness list. The changes in the list of security weaknesses proposed by SECURIFY indirectly confirmed the trend of recent security weaknesses and its importance. Except for one item, there is no change between the lists.

Table I. SECURIFY's Smart Contract Security Weakness List

\begin{tabular}{ccc}
\hline & Ver. 2018.06 & Ver. 2019.06 \\
\hline 1 & Transaction Reordering & Transaction Reordering \\
2 & Recursive Calls & Recursive Calls \\
3 & Insecure Coding Patterns & Insecure Coding Patterns \\
4 & Unexpected Ether Flows & Unexpected Ether Flows \\
5 & Use of Untrusted Inputs in Security & Dependence on unsafe inputs \\
\hline
\end{tabular}

\subsection{SMARTCHECK}

SmartCheck is a smart contract diagnostic tool which was developed by SmartDec in $2018[7,8]$. Table II shows the smart contract security weakness lists of SmartCheck. SmartCheck has made two recent changes to the original list.

Table II. SmartCheck's Smart Contract Security Weakness List

\begin{tabular}{lcc}
\hline & Ver. 2018.06 & Ver. 2019.06 \\
\hline 1 & No payable fallback function & No payable fallback function \\
2 & Reentrancy & Compiler version not fixed \\
3 & Unchecked math & Using tx.origin for authorization \\
4 & Unchecked low-level call & Unchecked low-level call \\
5 & Implicit visibility level & Implicit visibility level \\
\hline
\end{tabular}

\section{PROPOSED SECURE CODING RULES FOR ETHEREUM SMART CONTRACT}

In this study, we assess the security weaknesses that can occur while executing smart contracts on Ethereum. The security risks can be mitigated by diagnosing smart contracts and communicating the results to users based on the following items. Table III shows the common weakness enumeration (CWE) items that correspond to the items from the Ethereum smart contract security weakness list proposed in this study.

Table III. Common Weakness Enumeration (CWE) Items

\begin{tabular}{lll}
\hline \multicolumn{1}{c}{ Category } & \multicolumn{1}{c}{ Proposed Weakness } & \multicolumn{1}{c}{ Related CWE Item } \\
\hline Data validation & Integer Overflow and Underflow & CWE-682: Incorrect Calculation \\
\cline { 2 - 3 } Reentrancy & Unchecked Low Level Calls & $\begin{array}{l}\text { CWE-841: Improper Enforcement of } \\
\text { Behavioral Workflow } \\
\text { CWE-841: Improper Enforcement of } \\
\text { Behavioral Workflow }\end{array}$ \\
\cline { 2 - 3 } $\begin{array}{l}\text { Exception } \\
\text { Contract } \\
\text { characteristics } \\
\text { Special variables }\end{array}$ & DoS With Gas Limit & $\begin{array}{l}\text { CWE-400: Uncontrolled Resource } \\
\text { Consumption }\end{array}$ \\
\cline { 2 - 3 } & Multiple Inheritance & $\begin{array}{l}\text { CWE-696: Incorrect Behavior Order } \\
\text { CWE-284: Improper Access Control }\end{array}$ \\
\cline { 2 - 3 } & Authorization through tx.origin & CWE-477: Use of Obsolete Function \\
\hline
\end{tabular}




\subsection{INPUT DATA VALIDATION}

3.1.1. INTEGER OVERFLOW AND UNDERFLOW: Overflow is a flaw that causes errors or program security vulnerabilities by overwriting data in areas that should not be used, particularly if the process enters data that exceeds the maximum storage size. Underflow is when the user enters data that is smaller than the minimum size. This flaw is critical to software security because this error has plagued developers for decades. Solidity can handle up to 256-bit data when using unsigned integers. Hence, if the data value is increased, it will be 0 if it is greater than $2^{256}-1$, and if it is less than 0 , it will be $2^{256}-1$, which is incorrect, and it may be used [9-11]. Figure 1 shows an example of unsafe code for arithmetic operations.

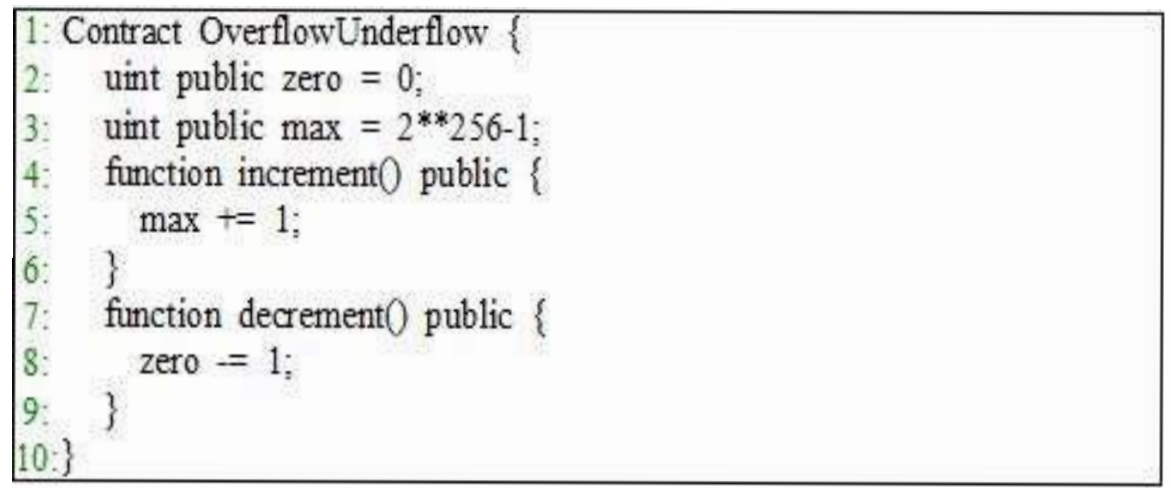

Fig. 1 Overflow and underflow example code

By using the exception throwing function assert() to check the boundary before using the data, the user can eliminate security weakness by invalidating the transaction that is likely to cause the flaw. Figure 2 shows an example of safe code for arithmetic operations.

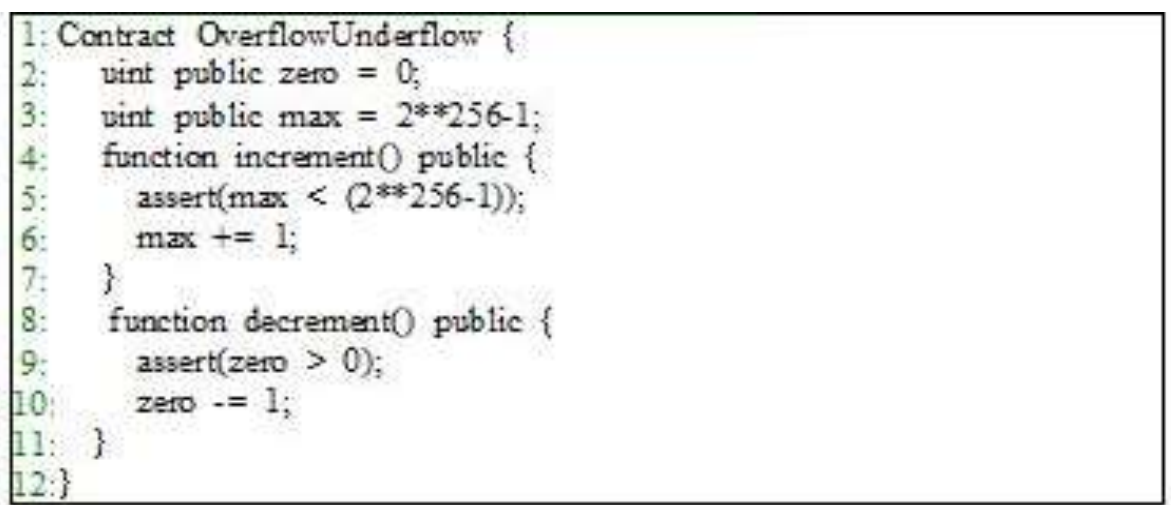

Fig. 2 Example of safe code for arithmetic operations

\subsection{REENTRANCY}

Invoking untrusted contracts can lead to unexpected risks or errors. An external call can execute malicious code using the untrusted contract or another contract that depends on that contract. Therefore, all external calls should be considered as potential security risks while programming.

3.2.1 ETHER TRANSFER FUNCTION: If the external call code is written inside the fallback function of the contract to transfer Ether, the following functionsaddress.call.value().Gas()(), address.send(), address.transfer() — can make an unexpected 
external call when used without considering the characteristics of each contract. First, address.call.value ().Gas()() specifies the gas to execute the fallback function's instructions, which can lead to unexpected code execution if malicious code is written. Next, address.send() limits the amount of gas the fallback function requires to execute the instruction. If the Ether transfer fails, it returns "false." Finally, address.transfer() also limits the gas volume but invalidates the transaction due to an exception when the Ether transfer fails. To execute the fallback command, the gas volume can be set via address.call.value().gas()(). Next, address.send() or address.transfer() is used so that the fallback function can handle only the Ether that was sent. However, since there is a risk of external calls within the fallback function, we aim to limit the transmission of gas. If the transfer fails, address.transfer() throws an exception and is safer than address.send() with respect to data protection. Hence, the Ether transfer function uses address.transfer(). Figure 3 shows an example of unsafe code for Ether transfer.

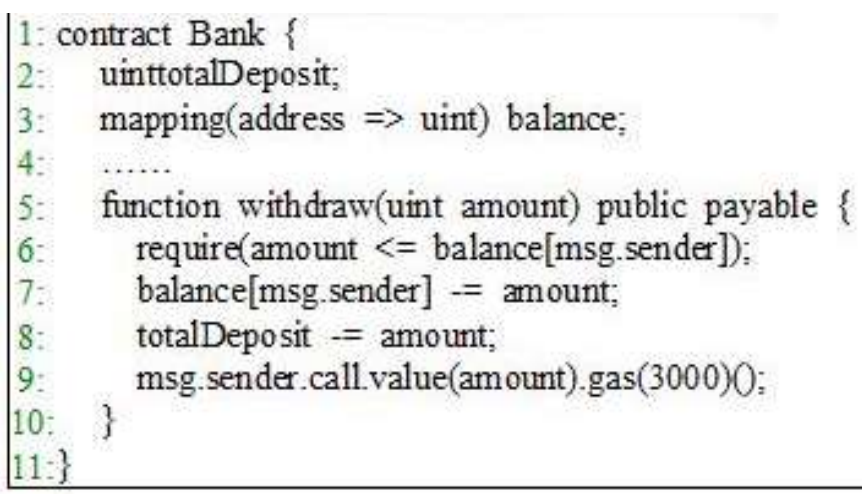

Fig. 3 Unsafe code example for Ether transport

Unexpected external calls may occur in the fallback function because it sets the amount of gas available when using address.call.value().Gas()(). External calls are a potential security risk because they can execute malicious code using the contract or by other contracts that depend on the contract. To prevent this, even if an attacker inserts the fallback function's malicious code and distributes the contract, it would be unexpected if the user sending the Ether could use address.transfer() to allow the fallback function to handle only the currently received Ether. Even if a security risk exists, it can be prevented [12]. Figure 4 shows an example of secure code for Ether transfer.

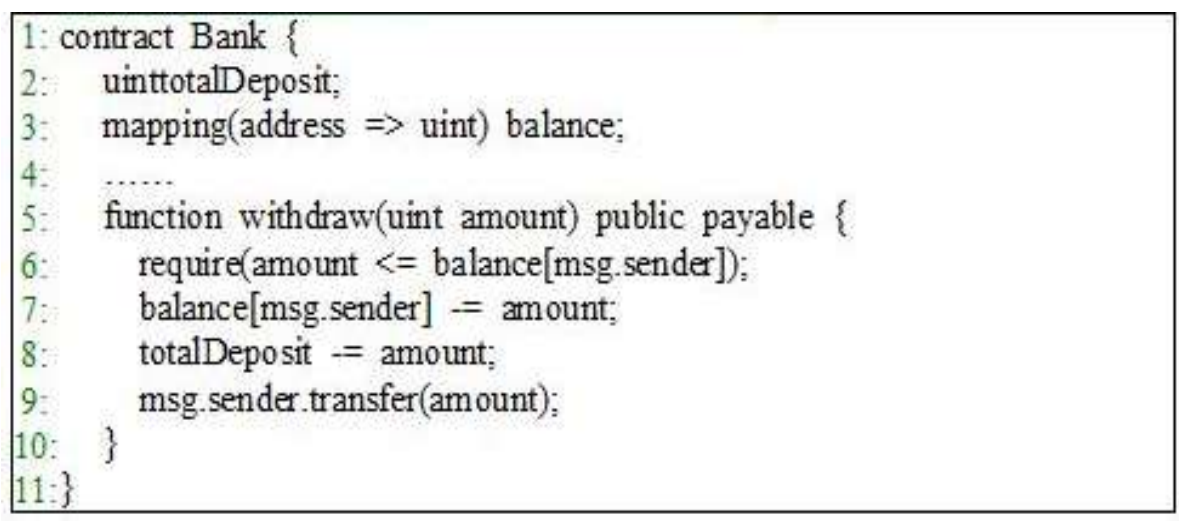

Fig. 4 Example of code safe for Ether transfer

3.2.2. FALLBACK FUNCTION: The fallback function is a function that is automatically called when the calling function does not exist in the contract or when it receives an Ether. If the user inserts an external calling code while implementing the 
fallback function, the attacker may pose an unexpected risk. Figure 5 shows an example of insecure code that functions as a fallback function.

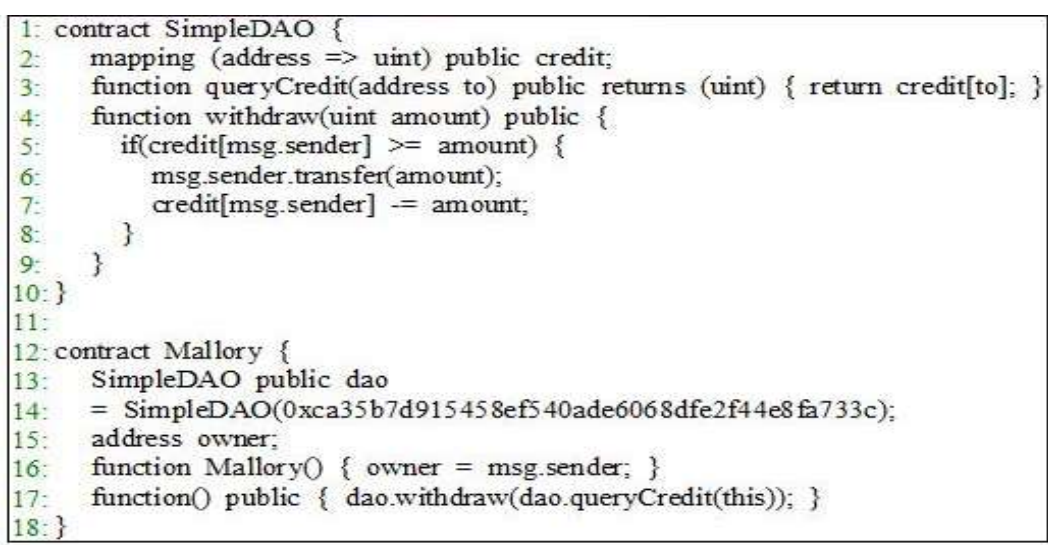

Fig. 5 Example of unsafe code with Fallback function

When sending Ether to the Mallory contract, the fallback function is called automatically and an external call is made internally through withdraw() of the SimpleDAO object. The function dao.withdraw() transfers Ether to the calling contract, which incurs a risk of reentrancy, in which the fallback function of the Mallory contract is called again [7]. If it is inevitable to insert an external calling code while writing a program, a mutex can be used. In the program shown in Figure 5, the withdraw() function raises an exception through require() when the lockCredit value is "true" so that the function is not executed and sets the lockCredit value to "false" just before the function ends. If the called function is a reentrant during execution, the lockCredit value is "true" to prevent reentry. The bool variable lockCredit value is checked with the called setup exception function require() to prevent reentrancy caused by external calls. Figure 6 shows an example code that prevents reentry of the fallback function.

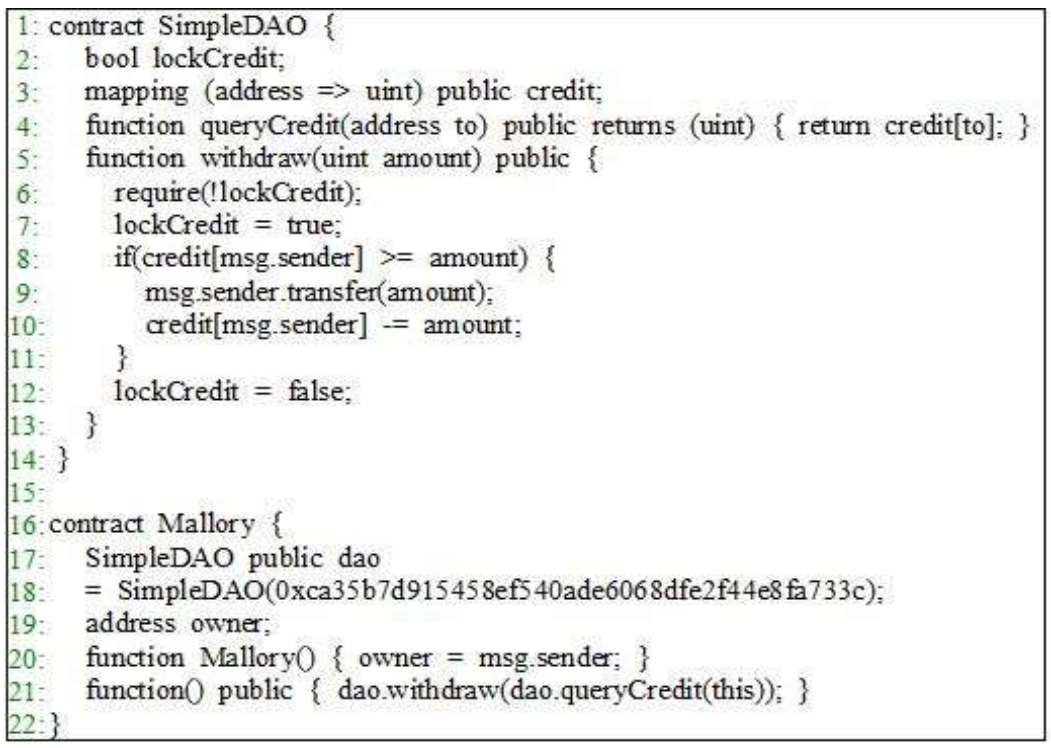

Fig. 6 Example code to prevent reentry of the fallback function

\subsection{EXCEPTION}

The exception is used to protect the data caused due to bad transactions. However, an attacker could use an exception code to prevent the contract from doing this. 
3.3.1 DENIAL OF SERVICE (DOS): Attacks that intentionally attack a system to prevent it from being used for its intended purpose can continue to generate exceptions. If an exception occurs, all transactions are invalidated. If a transaction for any purpose is always invalidated, it cannot be used for its intended purpose [13]. This can be prevented by separating the vulnerable parts under a DoS attack into separate transactions. Figure 7 shows a sample of DoS attacks.

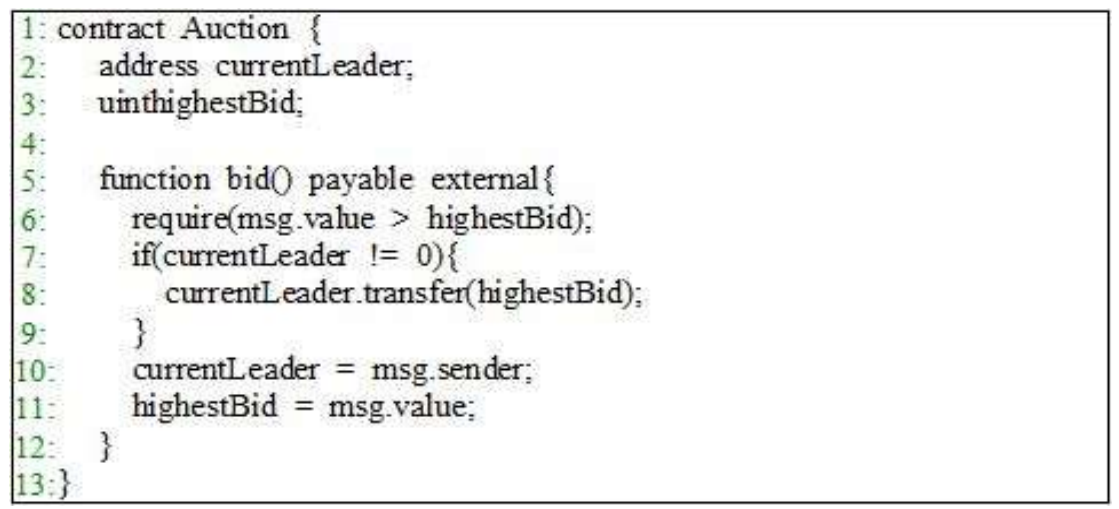

Fig. 7 Denial of Service attack example code

As shown in the sample code in Figure 7, when the highest bidder is changed, the Ether will be refunded to the address of the currentLeader. If transfer() fails - the account will be deleted after the bidding-the transaction will be invalid, and the bidding function cannot be used. To solve this problem, uint is stored as an address index in refunds using mapping. In the bid() function, if the bid is lower than the current highest bid, it is invalidated through require(). If not, the if statement stores the Ether to refunds to the previous best bidder. Thereafter, we assign the highest bidder's address to the currentLeader and change the highest bidder. If the top bidder changes, instead of automatically refunding the Ether to the previous top bidder, refund is initiated with withdrawRefund(), unlike bid(). Figure 8 shows an example of DoS prevention code.

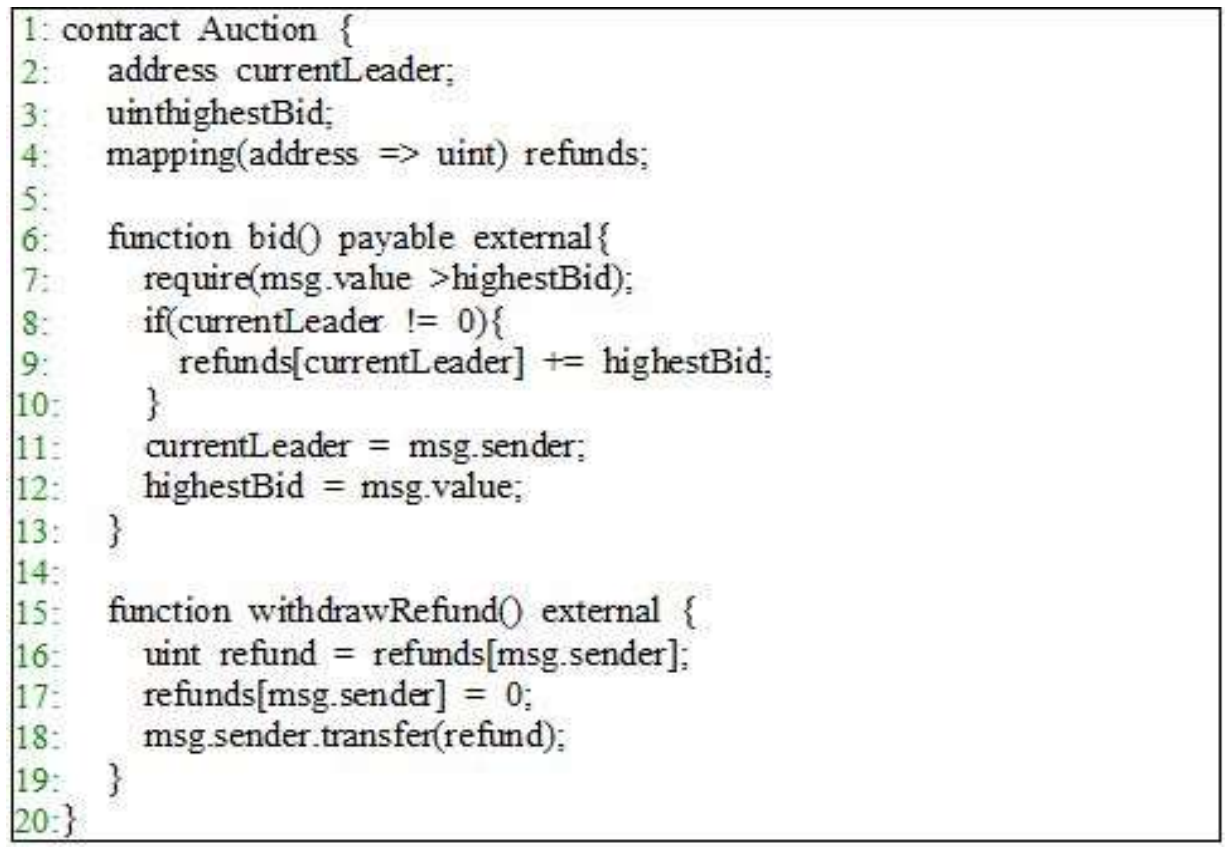

Fig. 8 Denial of service prevention example code 


\subsection{CONTRACT CHARACTERISTICS}

Solidity has object-oriented features. Just as classes are used in object-oriented languages such as $\mathrm{C}++$ and Java, Solidity abstracts contracts through contract specifiers and can hide information. Inheritance also enables code reuse and ensures polymorphism through redundancy and redefinition. However, this can lead to unexpected errors.

3.4.1. MULTIPLE INHERITANCE: Multiple inheritance is one of the object oriented programming features, and a class can inherit various behaviors or features from one or more super classes. Ambiguity can arise if there are member functions of the same name in multiple inheritance relationships [8]. Solidity handles the most recently inherited contract if ambiguity occurs in multiple inheritance relationships. This may be an unintended consequence, and Java does not support multiple inheritance of classes due to ambiguity issues. Multiple inheritance needs to be avoided to resolve ambiguities. Figure 9 shows an example code for multiple inheritance.

When the user calls set() after creating a final contract, the user gets a compile error considering the semantics of $\mathrm{C}++$. However, Solidity will call set() of BaseNo2 among set() overridden in BaseNo1 and BaseNo2, and the value of a will be 2 when get() is called. This can be a problem if the BaseNo1 contract contains important code. When using inheritance in Solidity, the compiler does not recognize it as an error, hence barring multiple inheritance to prevent unintended errors.

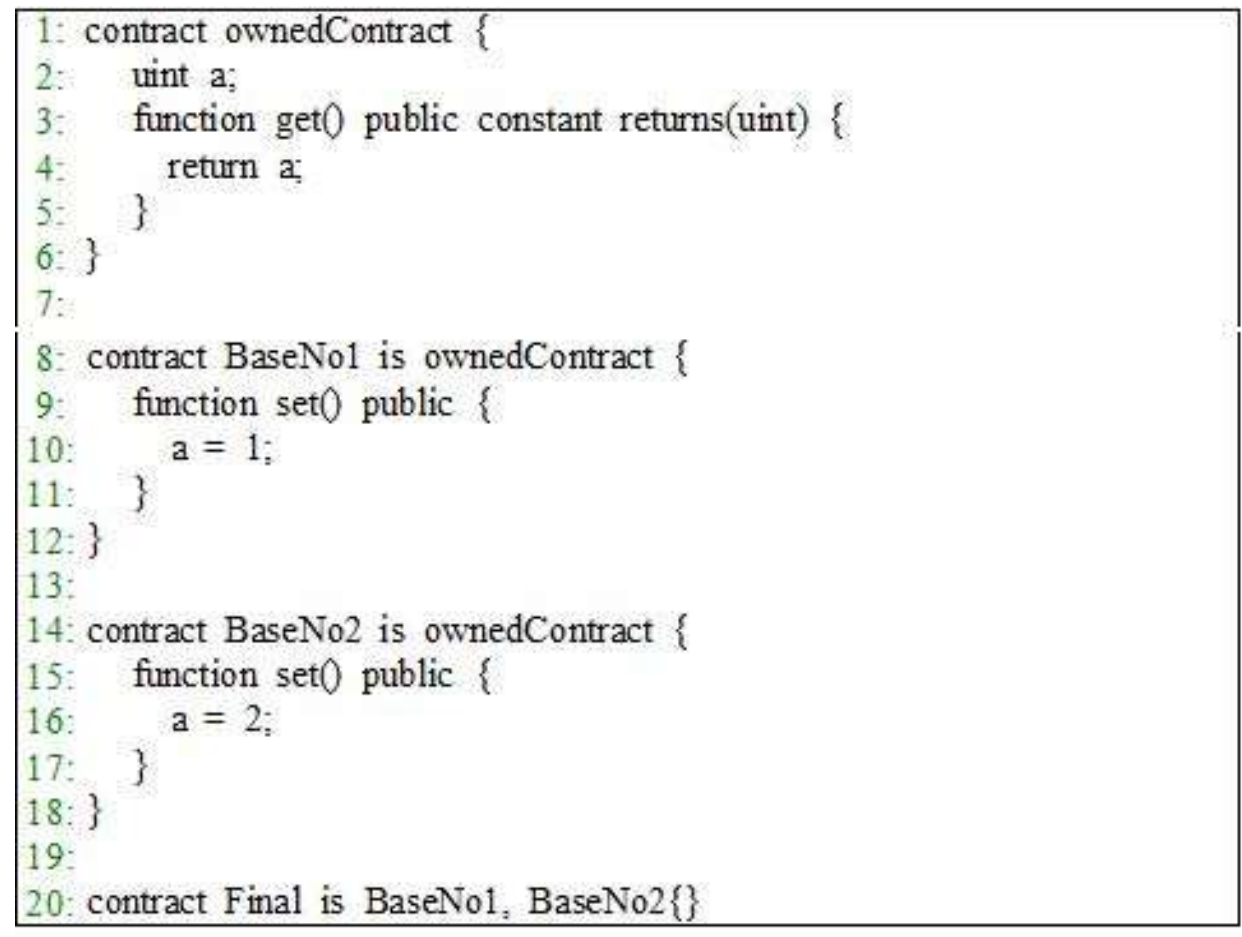

Fig. 9 Multiple inheritance example code

3.4.2. ACCESS SPECIFIER: Solidity defaults to the default accessor of contract internal variables, and the function is public. If the access specifier is set to default or used incorrectly, unexpected external access may be achieved. If the user does not explicitly specify an access specifier, the default specifier is applied. Since the function is public, anyone can access it, which can lead to unexpected risks [14]. Therefore, when designing program code, access specifier must be explicitly indicated. Figure 10 shows example code for using default access specifiers. 


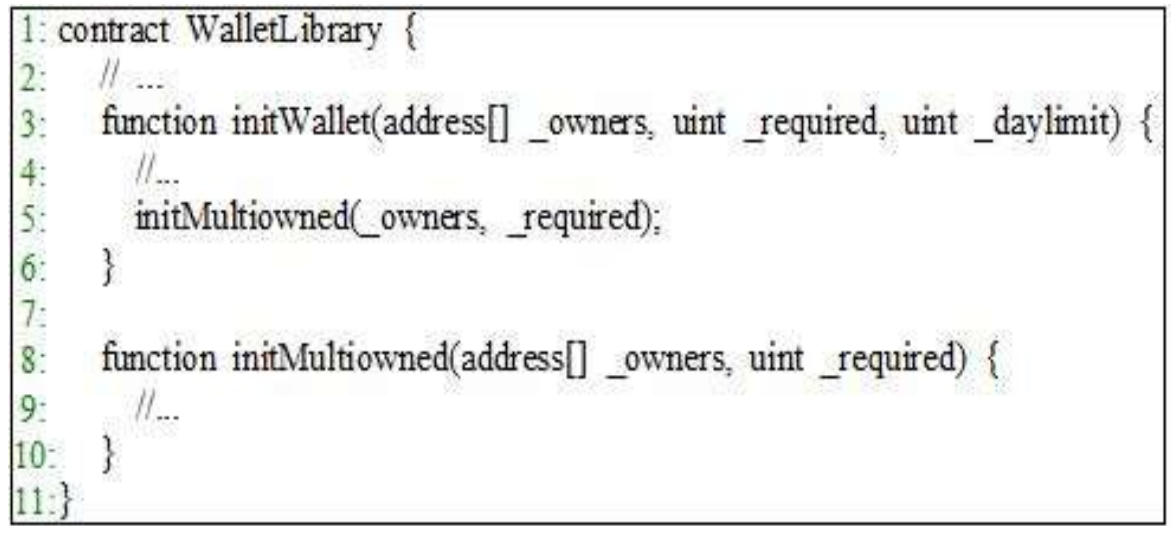

Fig. 10 Example code using default access specifier

As the main cause of the Parity wallet hacking incident, initWallet() should be called only by the owner of the wallet in the WalletLibrary. If the accessor of a function is public, anyone can access it. If it is internal, only a function can be accessed within the same contract. If the user sets an access specifier externally, the user can access the call or the direct transaction of the user account from a contract other than a function within the contract. Figure 11 shows an example of using explicit access specifiers, which uses internal to make them externally inaccessible.

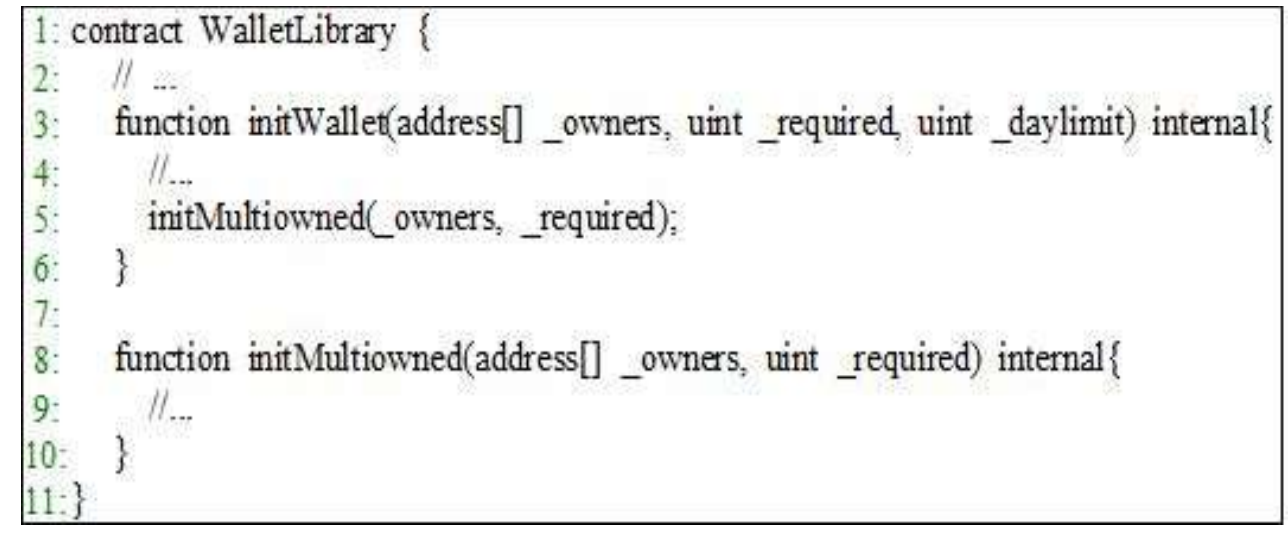

Fig. 11 Example code using explicit specifiers

\subsection{SPECIAL VARIABLES}

In Solidity, there are always special variables in the global namespace that provide information about the blockchain. Though these variables make it easy to access the required data, security weaknesses also exist.

3.5.1. $t \boldsymbol{x}$ VARIABLE: Programmers can easily get the data they need using tx, using functions such as tx.gasprice and tx.origin, which holds data about transactions. where, gasprice has the transaction's gas price and origin has the transaction's original producer address. However, the distribution of malicious contracts using this data can pose unexpected risks [15]. Figure 12 shows example code for using tx.orign. 


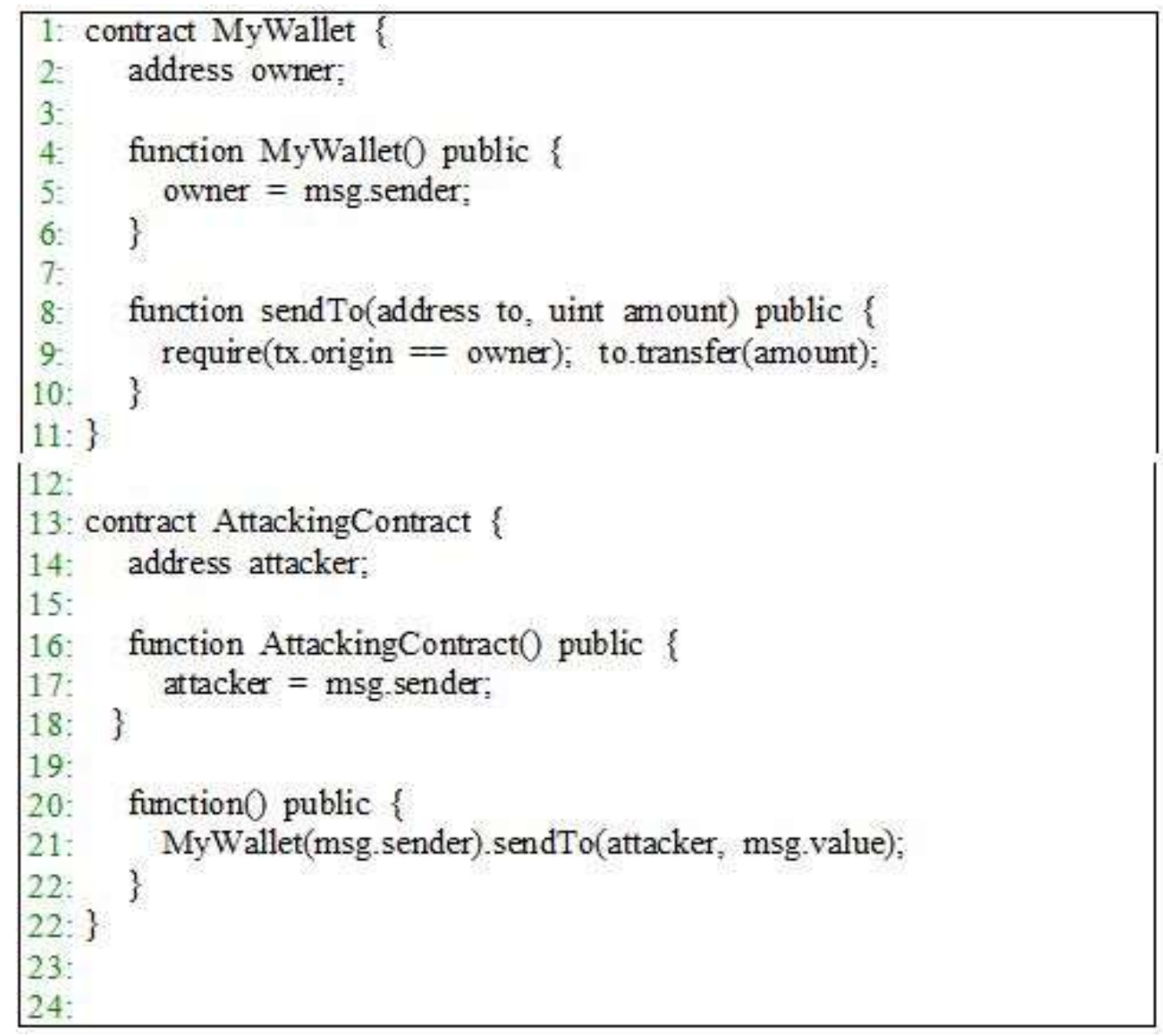

Fig. 12 Example code using tx.orign

When the user sends Ether from the wallet to the AttackingContract, the fallback function is executed automatically and calls MyWallet(). The function SendTo() passes the constructor address of the attacker and the Ether of the current transaction as parameters. In MyWallet, the owner value and the tx.origin value are the user's address. Eventually, the owners of tx.origin and MyWallet will match. Therefore, the call to require () does not throw an exception and sends Ether to address to. to is the address of the attacker and the user may lose the transfer Ether.

In the example code in Figure 12, the programmer tried to compare the user with the constructor that deployed the contract using tx.origin. However, unexpected error may arise if the contract generates the contract. To prevent this error, use of tx.origin should be avoided. The origin address can be obtained by connecting the address that the user originally distributed the contract to as a parameter. Figure 13 shows an example of using a secure home address. 


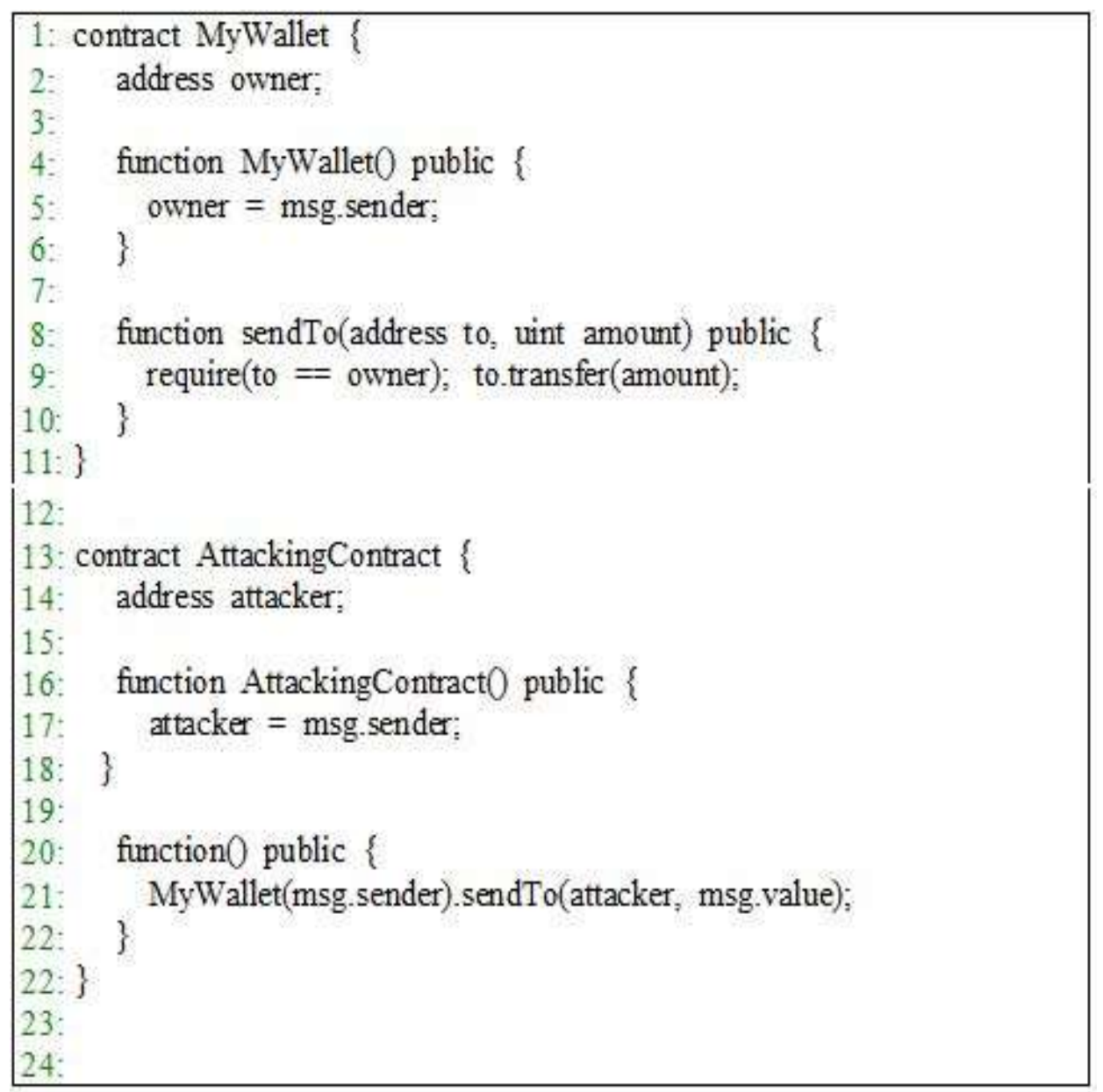

Fig. 13 Example code using a secure origin address

\section{CONCLUSION AND FURTHER STUDIES}

As expectations from blockchain-based smart contracts grow, their use is increasing. However, an unexpected bug in running an insecure smart contract can make it vulnerable to security issues. Therefore, for the reliable use of smart contracts, security weakness items need to be created and diagnosed.

In this paper, we assessed the vulnerabilities in the security of Ethereum smart contracts. Security weakness diagnosis can enhance the security of smart contracts. In the future, we plan to develop a diagnostic tool that can automatically diagnose the security weaknesses presented in this paper.

\section{ACKNOWLEDGEMENTS}

This research was supported by Basic Science Research Program through the National Research Foundation of Korea (NRF) funded by the Korea Government (MSIT) (No. 2017R1C1B5018257), by the National Research Foundation of Korea (NRF) grant funded by the Korea Government (MSIT) (No. 2018R1A5A7023490) and by the National Research Foundation of Korea (NRF) grant funded by the Korea Government (MSIT) (No.2019R1F1A1045343). Also, this research was supported by the Basic Science Research Program through the National Research Foundation of Korea (NRF) funded by the Ministry of Education (No. 2017R1D1A3B03029906). 


\section{REFERENCES}

[1] M. I. Mehar, C. L. Shier, A. Giambattista, E. Gong, G. Fletcher, R. Sanayhie, H. M. Kim, M. Laskowski, "Understanding a revolutionary and flawed grand experiment in blockchain: the DAO attack," Journal of Cases on Information Technology, Vol. 21, No. 1, (2019), pp. 19-32.

[2] The Parity Wallet Hack Explained, https://blog.zeppelin.solutions/on-the-parity-wallet-multisig-hack405a8c12e8f7

[3] G. Destefanis, M. Marchesi, M. Ortu, R. Tonelli, A. Bracciali, R. Hierons, "Smart contracts vulnerabilities: a call for blockchain software engineering," 2018 International Workshop on Blockchain Oriented Software Engineering (IWBOSE), (2018), pp. 19-25.

[4] T. Abdellatif, K. L. Brousmiche, "Formal verification of smart contracts based on users and blockchain behaviors models," 2018 9th IFIP International Conference on New Technologies, Mobility and Security (NTMS), (2018), pp. 1-5.

[5] SECURIFY, https://securify.ch

[6] P. Tsankov, A. Dan, D. Drachsler-Cohen, A. Gervais, F. Buenzli, M. Vechev, "Securify: Practical security analysis of smart contracts," Proceedings of the 2018 ACM SIGSAC Conference on Computer and Communications Security, (2018), pp. 67-82.

[7] SmartCheck, https://tool.smartdec.net

[8] S. Tikhomirov, E. Voskresenskaya, I. Ivanitskiy, R. Takhaviev, E. Marchenko, Y. Alexandrov, "Smartcheck: Static analysis of ethereum smart contracts," 2018 IEEE/ACM 1st International Workshop on Emerging Trends in Software Engineering for Blockchain, (2018), pp. 9-16.

[9] K. Delmolino, M. Arnett, A. Kosba, A. Miller, E. Shi, "Step by step towards creating a safe smart contract: Lessons and insights from a cryptocurrency lab," International Conference on Financial Cryptography and Data Security, (2016), pp.79-94.

[10] K. Bhargavan, A. Delignat-Lavaud, C. Fournet, A. Gollamudi, G. Gonthier, N. Kobeissi, A. Rastogi, T. Sibut-Pinote, N. Swamy, S. Zanella-Béguelin, "Formal verification of smart contracts: Short paper," Proceedings of the 2016 ACM Workshop on Programming Languages and Analysis for Security, (2016), pp. 91-96.

[11] Y. Lee, J. Jeong, Y. Son, "Design and implementation of the secure compiler and virtual machine for developing secure IoT services," Future Generation Computer Systems, Vol. 76, (2017), pp. 350-357.

[12] Ethereum Smart Contract Best Practices, Recommendations for Smart Contract Security in Solidity, https://consensys.github.io/smart-contract-best-practices/recommendations/

[13] Solidity 0.4.21 documentation, https://solidity.readthedocs.io/en/v0.4.21/contracts.html

[14] Known Attacks, https://consensys.github.io/smart-contract-best-practices/known_attacks/

[15] E. Zhou, S. Hua, B. Pi, J. Sun, Y. Nomura, K. Yamashita, H. Kurihara, "Security assurance for smart contract," 2018 9th IFIP International Conference on New Technologies, Mobility and Security (NTMS), (2018), pp. 1-5. 
International Journal of Advanced Science and Technology Vol.133 (2019) 